
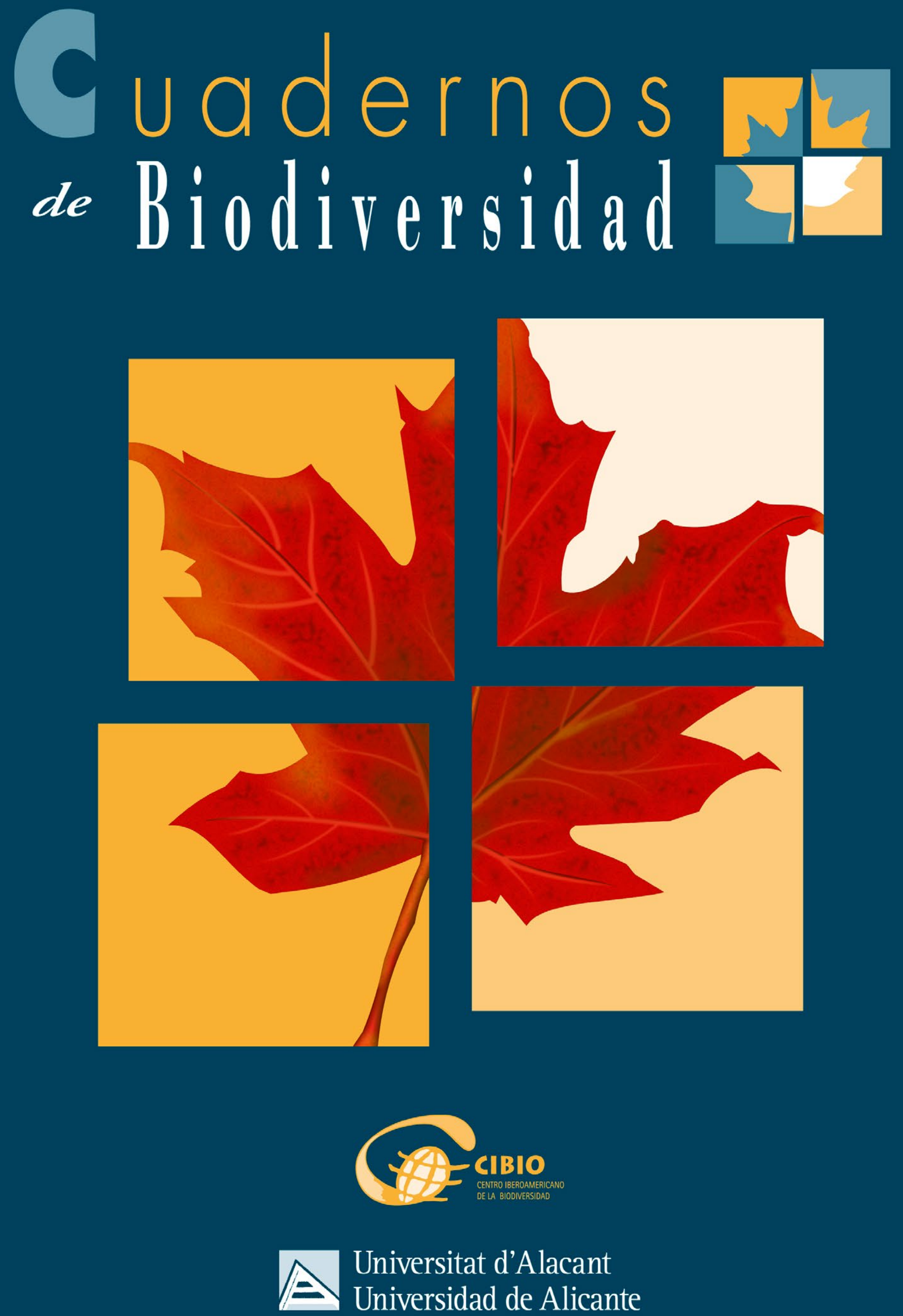


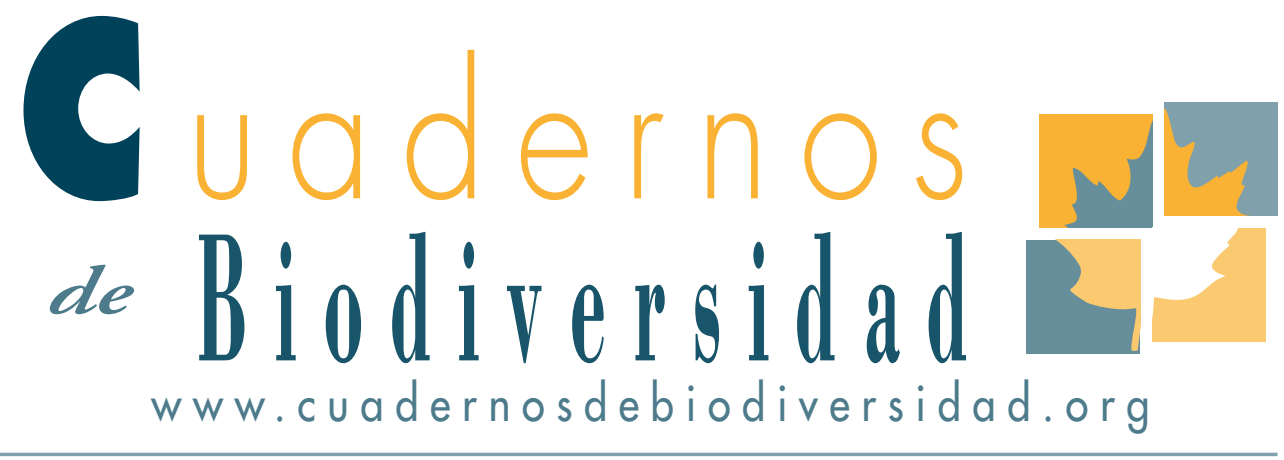

\section{Zamia inermis, \\ la cícada más amenazada de México}

\section{Zamia inermis, \\ the more threatened cycad of Mexico}

\section{P. Octavio-Aguilar ${ }^{1}$, L. G. Iglesias-Andreu ${ }^{2 *}$, A.P. Vovides ${ }^{3}, Y$ A. Rivera-Fernándeź .}

i Laboratorio de Genética, Centro De Investigaciones Biológicas, Instituto de Ciencias Básicas e Ingeniería, Universidad Autónoma del Estado de Hidalgo, Pachuca, Hidalgo, MÉXICO.

2*Instituto de Biotecnología y Ecología Aplicada (Inbioteca). Universidad Veracruzana Avenida de las Culturas Veracruzanas io i, Colonia Emiliano Zapata, C.P. 9iogo, Xalapa, Ver., México. Correspondence Author: liglesias@uv.mx

3 Laboratorio De Biología Evolutiva De Cycadales, Jardín Botánico Fco. Javier Clavijero, Campus II, Instituto de Ecología A. C. Xalapa, Veracruz, México.

4 Facultad De Ciencias Agrícolas, Universidad Veracruzana, Xalapa, Veracruz México.

\section{RESUMEN}

Zamia inermis (Zamiaceae) es una cícada rara, endémica y actualmente listada en Peligro Crítico de Extinción. De la misma, sólo se conoce una población compuesta de tres subpoblaciones, que se encuentra distribuido en tan sólo $2,5 \mathrm{~km} 2$ de pastizales y bosques estacionalmente secos y fragmentados. Durante la década de los 70's y 80 s se sustrajeron ilegalmente muchas plantas para comercio, por lo que su población actual estimada está compuesta entre 300 y 700 ejemplares. Además, debido a la ausencia de su polinizador natural, un escarabajo, esta especie presenta un reclutamiento extremadamente bajo y su diversidad genética está insuficientemente representada en esta singular población. Por todas estas razones se le considera como las cícada mexicana que se encuentra en mayor riesgo de extinción.

Palabras clave: cícadas, especies en peligro crítico de extinción, conservación, manejo. 


\section{ABSTRACT}

Zamia inermis (Zamiaceae) is a rare endemic and critically threatened species of cycad, with only one known population, composed of three subpopulations. The total population is distributed in only $2.5 \mathrm{~km} 2$ on grassland and fragmented seasonally dry forest. Many plants have been illegally removed for the ornamental plant trade during the 1970's and 80 s and its current population is estimated between 300 and 700 individuals, with extremely poor recruitment due to absence of its natural beetle pollinator. Additionally, its genetic diversity is under represented in this unique population, and for these reasons this species should be considered as the Mexican cycad at greatest extinction risk.

Key words: Cycads, critically endangered species, conservation, management.

\section{INTRODUCCIÓN}

Las cícadas son plantas longevas surgidas hace más de 200 ma con una extensa radiación a lo largo de las zonas tropicales y subtropicales del planeta. Las especies americanas son más recientes y cuentan con una mayor diversidad, destacando particularmente los géneros hermanos Ceratozamia, Zamia y Microcycas, este último endémico a Cuba (Nagalingum et al., 2011, Salas-Leiva et al., 2013, Codamine et al., 2015). El género Dioon, siendo prácticamente endémico a México y considerado ancestral en el nuevo mundo al igual que cycas en el viejo mundo; cuenta con alrededor de 14 especies incluyendo una planta hondureña. Es el género más espectacular pues alcanza alturas superiores a los $10 \mathrm{~m}(D$. spinuloum, y D. rzedowski de Oaxaca y D. mejiae de Honduras), además pueden llegar a vivir más de 2000 años (Vovides \& Peters, 1987). En la costa del Golfo de México se ha descrito la presencia de 17 especies pertenecientes a los géneros Ceratozamia, Dioon y Zamia; de las cuales cinco (Ceratozamia huastecorum, C. morettii, Zamia furfuracea, $Z$. inermis y $Z$. vazquezii) se desarrollan endémicamente en las regiones centro y sur del estado de Veracruz, México.
No obstante, la mayoría de las poblaciones de cícadas han sido durante años afectadas por diversas actividades antropogénicas, extracción ilegal, cambio de uso de suelo, fragmentación del hábitat y por el uso de agroquímicos. Especialmente en el caso de $Z$. inermis, donde los agroquímicos matan a los polinizadores, así como en Microcycas calocoma de Pinar del Río, Cuba, en la que sucede algo similar (Vovides, 1989, Vovides et al., 1997). Ante este panorama, aun cuando hay extensos trabajos para la conservación y manejo de estas especies en invernaderos y viveros rurales (Vovides et al., 2002), varias cícadas se encuentran listadas en el libro rojo de la Unión Internacional para la Conservación de la Naturaleza en la categoría Critically Endangered (CR).

Hoy en día, 10 de las 55 especies de cícadas reportadas para México se consideran en extremo peligro de extinción. Entre ellas están: Ceratozamia euryphyllidia, distribuida en Oaxaca y Veracruz (probablemente extinta de Veracruz); C. fuscoviridis, distribuida en Hidalgo y probablemente en San Luis Potosí y Puebla; C. kuesteriana, propia de Tamaulipas y San Luis Potosí; C. norstogii, procedente de Chiapas; C. zaragozae, endémica de San Luis Potosí; Dioon caputoi, distribuida en Puebla y Oaxaca; Zamia inermis, endémica de Veracruz; $Z$. variegata, distribuida en Chiapas, Belice y Guatemala; $Z$. spartea, propia de Chiapas y Oaxaca; así como $Z$. vazquezii, de Hidalgo y Veracruz (IUCN, 2016).

Recorridos exhaustivos efectuados en las zonas antes mencionadas han permitido detectar la presencia de nuevas poblaciones. Por ejemplo, de $C$. zaragozae, que originalmente fue descrita a partir de una única población en el norte de San Luis Potosí y contaba únicamente con 50 individuos en estado natural, hoy se tienen registradas seis poblaciones naturales con un número aproximado de 5000 individuos (Castillo-Lara, com,. Pers.) gracias a los esfuerzos realizados durante más de 10 años por parte de los investigadores del Instituto de Zonas Desérticas de la Universidad Autónoma de San Luis Potosí. De igual manera, $C$. fuscoviridis, recién redescubierta (Osborne et al., 2006) y que fue descrita a partir de una única población en el centro de Hidalgo, hoy 
en día cuenta con más de 28 registros y algunos reportes incidentales en Puebla y San Luis Potosí (Pulido et al., 2015). Asimismo para Dioon caputoi solo se conocían dos poblaciones donde persistían 750 plantas (Cabrera-Toledo et al., 2012), hoy en día se han localizado más de 5 poblaciones en las inmediaciones de Oaxaca y Puebla. Bajo este panorama, es muy posible que la escasez de individuos que surge ante una distribución estrecha y pocos reportes se deba más bien a una deficiencia en los trabajos de prospección y muestreo. Sin embargo, el caso de Zamia inermis escapa a esta condición.

\section{ESTADO ACTUAL DE LAS POBLACIONES DE Z. INERMIS}

Zamia inermis fue descubierta en un predio particular en 1973 y no fue hasta 1983 cuando se describió formalmente (Vovides et al., 1983). Para entonces cientos, quizá miles de plantas ya habían sido extraídas con fines de comercialización. Durante años se han realizado extensivos recorridos en la Sierra de Manuel Díaz (Fig. 1), en Monzomboa, Veracruz, ubicando únicamente tres subpoblaciones en menos de $2.5 \mathrm{~km} 2$ con un total de 654 individuos, la mayoría de los cuales son adultos no reproductivos.

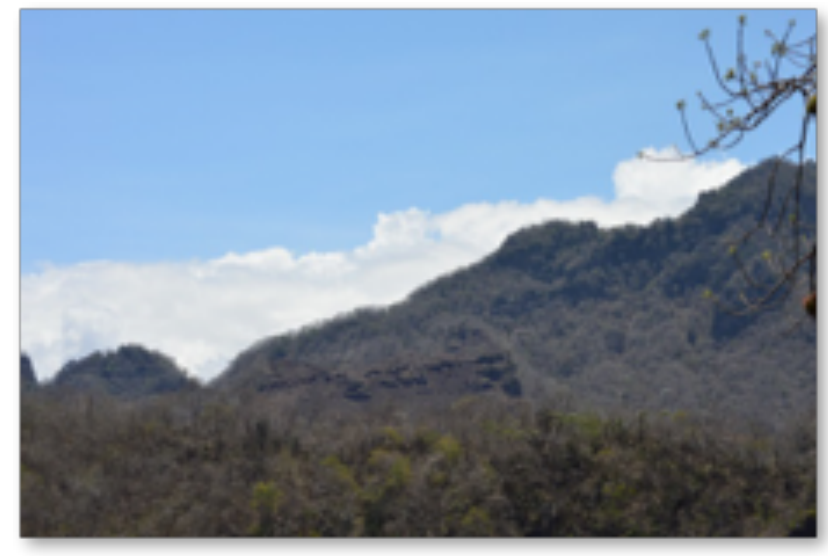

Figura 1. Vista panorámica de la población de Zamia inermis en la Sierra de Manuel Díaz, Monzomboa, Veracruz, México (Foto: Zavaleta, 2015)
Se ha encontrado además que la viabilidad de las semillas ha disminuido sustancialmente en los últimos 20 años ya que de un 100\% de germinación que se obtenía a partir de semillas colectadas en campo hoy sólo obtiene un $10 \%$. De las pocas plantas germinadas en traspatios a día de hoy sólo sobreviven 92, por lo que este tipo de propagación resulta ineficiente para la conservación de la especie (Sánchez-Tinoco et al., 1993). Esta situación probablemente refleja un mal manejo hortícola, ya que periódicamente se han llegado a obtener semillas viables a partir de polinización manual hasta alcanzar la talla de adultos en diferentes viveros (Broome, com. pers.).

Sin embargo, a pesar de que los pobladores siguen esforzándose para propagar las pocas semillas encontradas en campo logrando ocasionalmente alguna germinación, el problema principal sigue siendo la falta del agente polinizador y la consecuente baja viabilidad de semillas. Por ello resulta de gran importancia la implementación de técnicas de polinización manual. Sin embargo, para ello se requeriría establecer un programa de retribución económica a los pobladores interesados en participar en dicha actividad.

A pesar de que desde hace 11 años un $19.87 \%$ de las plantas, tanto machos como hembras, han presentado eventos reproductivos, la mayoría de las semillas producidas son infértiles y no se ha podido encontrar ningún polinizador en los conos masculinos. Ante este panorama, las pocas plántulas (Fig. 2) que se han localizado en la población parecen depender espacialmente de la presencia de machos en radios menores a los tres metros, lo que resulta sumamente ineficiente en comparación a lo que se ha observado en otras poblaciones naturales de cícadas (Norstog et al., 1986). Esta situación sugiere que el polen es un recurso escaso, o no se encuentra disponible en la población. Este escenario se complica por el hecho de que prácticamente todas las plántulas en campo mueren antes de alcanzar un año, por lo que la tasa de crecimiento muestra un claro descenso en el tamaño poblacional. 


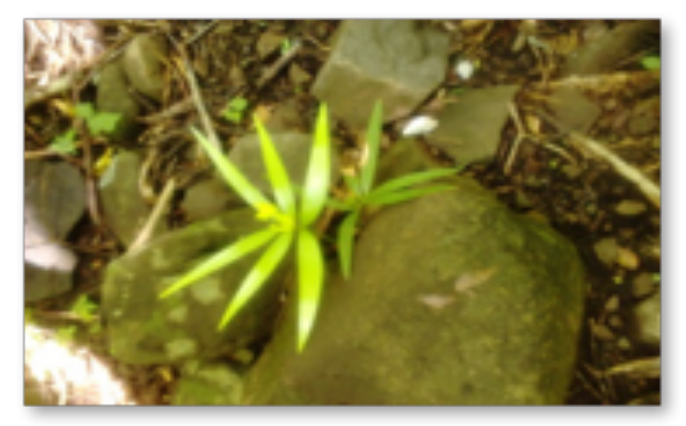

Figura 2. Plántula de $Z$. inermis en su hábitat natural, Monzomboa, Veracruz, México.

(Foto: Zavaleta, 2015)

Para tener un panorama más claro sobre el estado de conservación de las poblaciones de $Z$. inermis, es necesario identificar el potencial adaptativo de la especie, relacionado de manera directa con la diversidad genética, ya que una mayor variabilidad garantiza una respuesta más amplia ante diferentes problemas evolutivos (Lande, 1988). Al respecto, la medida más utilizada para evaluar la variabilidad en las poblaciones es la heterocigosis. Para esta especie, este valor resulta estar por debajo del promedio para la mayoría de las gimnospermas (Hamrick et al., 1992) y del resto de las cícadas, lo cual la ubica en el rango de las especies del viejo mundo y bastante abajo de la diversidad genética para las cícadas del nuevo mundo (González-Astorga et al., 2009). Además, las 92 plantas cultivadas en traspatios presentan un valor de heterocigosis mucho menor que lo observado en la población natural, por lo que no resultan representativas de la variabilidad de la especie, con lo cual esta práctica de manejo está generando una pérdida de diversidad. Por todo lo anterior, se podría afirmar que Zamia inermis es la cícada más amenazada de México y probablemente de toda América Latina.

\section{PROPUESTAS DE CONSERVACIÓN}

El panorama general para la especie es desalentador pues, de acuerdo a las proyecciones demográficas y genéticas, la especie podría extinguirse en condiciones naturales en los próximos 70 años, si se considera que los individuos llegasen a alcanzar su etapa reproductiva entre los tres y los ocho años (Octavio-Aguilar, com. pers.). Por ello, los esfuerzos de conservación para esta especie se han centrado en tres aspectos generales:

a) Polinización manual: Esta técnica ha mostrado ser eficiente en diferentes jardines botánicos así como en colecciones privadas. Para ello se puede usar polen suspendido en soluciones acuosas que se inyectan directamente en el megasporófilo (cono femenino) o con polen seco proyectado por presión de aire directamente en los conos receptivos. Actualmente, esta técnica se está llevando a cabo en el Jardín Botánico "Clavijero", en la ciudad de Xalapa, Veracruz; aunque a nivel de campo resultaría insuficiente o privativo en cuanto a tiempo, recursos e interés por parte de los pobladores y sus autoridades.

b) Propagación in vitro: A partir de las técnicas de cultivo de tejidos se puede lograr la micropropagación de esta especie e incrementar la cantidad de individuos viables para garantizar restaurar la población. Esta técnica pudiera resultar una alternativa promisoria si se tiene en cuenta que la variación somaclonal asociada con estrategias de micropropagación de individuos genéticamente diversos podría contribuir a ampliar la diversidad genética de la especie. Además de que las herramientas biotecnológicas pudieran contribuir a conservar este valioso recurso genético. Estas técnicas se están implementando en los laboratorios del Instituto de Biotecnología y Ecología Aplicada de la Universidad Veracruzana y en el jardín botánico de la UNAM, aunque todavía se cuentan con resultados incipientes.

c) Germinación asistida: En general las cícadas tienen una germinación elevada en cultivo, siempre que se promuevan las condiciones adecuadas de riego y sustrato. En el Instituto de Biotecnología y Ecología Aplicada y en la Facultad de Agronomía de la Universidad Veracruzana se han estado realizando diferentes experimentos para incrementar el porcentaje de germinación de diversas especies de cícadas, con resultados verdaderamente prometedores. 
Finalmente, mientras se continúen empleando plaguicidas en las zonas adyacentes a la población nativa, se seguirá afectando a los polinizadores, con lo cual se mantendrá la tendencia actual de decline de la especie. Puesto que el uso del suelo en el predio donde se encuentra la planta es netamente agrícola, es recomendable efectuar la reubicación de la mayoría de los individuos a zonas conservadas simpátricas con otras cícadas que presenten poblaciones saludables de polinizadores, o se podría establecer un área natural protegida en el predio en cuestión. En conjunto, todas estas acciones podrían ser implementadas simultáneamente para garantizar la supervivencia de Zamia inermis.

\section{AGRADECIMIENTOS}

A CONACYT por el apoyo financiero brindado al Proyecto de Ciencia Básica 152073

\section{REFERENCIAS}

Cabrera-Toledo, D., González-Astorga, J. \& Flores-Vázquez, J.C. (2012). Fine-scale spatial genetic structure in two Mexican cycads species Dioon caputoi and Dioon merolae (Zamiaceae, Cycadales): implications for conservation. Bioch Sys Ecol, 40:43-48. Doi.10.1016/j. bse.2011.09.004.

Codamine, F.L., Nagalingum, N.S., Marshall, C.R. \& Morlon, H. (2015). Origin and diversification of living cycads: a cautionary tale on the impact of the branching process prior in Bayesian molecular dating. BMC Evol Biol, 17:15-65. Doi. 10.1186/s12862-015-0347-8

González-Astorga, J., Vovides, A.P., Cabrera-Toledo, D. \& Nicolalde-Morejón, F. (2009). Diversity and genetic structure of the endangered cycad Dioon sonorense (Zamiaceae) from Sonora, Mexico: Evolutionary and conservation implications. Biochem Sys Ecol, 36:891-899. Doi.10.1016/j.bse.2008.11.006

Hamrick, J.L., Godt, M.J.W. \& Sherman-Broyles, S.L. (1992). Factors influencing levels of genetic diversity in woody plant species. New Forest, 6:95-124. Doi.10.1007/ BF00120641

IUCN. (2016). Red list of threatened species of the International Union for Conservation of Nature. www.iucnredlist.org. Accedido: 18 May 2016.
Lande, R. (1988).Genetics and demography in biological conservation. Science, 241:1455-1460. Doi. 10.1126/ science.3420403

Nagalingum, N.S., Marshall, C.R., Quental, T.B., Rai, H.S., Little, D.P. \& Mathews, S. (2011). Recent synchronous radiation of a living fossil. Science, 334:796-799. Doi. 10.1126/science. 1209926

Norstog, K.J., Stevenson, D.W. \& Niklas, K.J. (1986). The role of beetles in the pollination of Zamia furfuracea $\mathrm{L}$. fil. (Zamiaceae). Biotropica, 18:300-306.

Osborne, R., Stevenson, D. \& Vovides, A.P. 2006. What is Ceratozamia fuscoviridis? Delpinoa, 48: 5-10.

Pulido, M.T., Vargas-Zenteno, M., Vite, A. \& Vovides, A.P. (2015). Range extension of the endangered Mexican cycad Ceratozamia fuscoviridis Moore (teosinte): implications for conservation. Trop Cons Sci, 8(3):778-795.

Salas-Leiva, D.E., Meerow, A.W., Calonje, M., Griffith, M.P., Francisco-Ortega, J., Nakamura, K., Stevenson, D.W., Lewis, C.E. \& Namoff, S. (2013). Phylogeny of the cycads based on multiple single-copy nuclear genes: congruence of concatenated parsimony, likelihood and species trees inference methods. Ann Bot, 112(7):12631278. Doi. 10.1093/aob/mct192.

Sánchez-Tinoco, M.Y., Vázquez-Torres, S.M. \& Cruz-Kuri, L. (1993). Determinación del dimorfismo sexual en Zamia inermis Vovides, Rees \& Vázquez-Torres, Zamiaceae (Cycadales), basado en características morfológicas vegetativas. La Ciencia y el Hombre, 15: 113-127.

Vovides, A.P., Rees, J. \& Vázquez-Torres, S.M. (1983). Flora de Veracruz. Zamiaceae. Fascículo 26. INIREB, Xalapa, Veracruz, México.

Vovides, A.P. (1989). Problems of endangered species conservation in Mexico: Cycads an example. Encephalartos, 20:29-35.

Vovides, A. P., Ogata, N., Sosa, V. \& Peña-García, E. (1997). Pollination of the endangered Cuban cycad Microcycas calocoma (Miq.) A.DC. Bot J Linn Soc, 125: 201-210. Doi. 10.1111/j.1095-8339.1997.tb02254.x

Vovides, A.P. \& Peters, C.M. (1987). Dioon edule: la planta más antigua de México. Ciencia y Desarrollo, XIII (73): 19-24.

Vovides, A.P., Iglesias, C., Pérez-Farrera, M.A., Vázquez-Torres, S.M. \& Schippmann, U. (2002). Peasant nurseries: A concept for an integrated conservation strategy for cycads in Mexico. In: Maunder, M., Clubbe, C., Hankamer, C. \& Groves, M. (eds.), Plant Conservation in the Tropics: Perspectives and Practice. Richmond, Royal Botanic Gardens, Kew. pp. 421-444. 